\title{
Enseignement du français et bilinguisme en Tunisie
}

Ahmed Ben Jemia

\section{OpenEdition}

\section{Journals}

Édition électronique

URL : http://journals.openedition.org/ries/2863

DOI : $10.4000 /$ ries.2863

ISSN : 2261-4265

\section{Éditeur}

Centre international d'études pédagogiques

\section{Édition imprimée}

Date de publication : 1 septembre 1998

Pagination : 49-52

ISSN : 1254-4590

\section{Référence électronique}

Ahmed Ben Jemia, «Enseignement du français et bilinguisme en Tunisie », Revue internationale d'éducation de Sèvres [En ligne], 19 | septembre 1998, mis en ligne le 18 avril 2013, consulté le 23 mars 2021. URL : http://journals.openedition.org/ries/2863 ; DOI : https://doi.org/10.4000/ries.2863

Ce document a été généré automatiquement le 23 mars 2021.

(c) Tous droits réservés 


\title{
Enseignement du français et bilinguisme en Tunisie
}

\author{
Ahmed Ben Jemia
}

1 Depuis la dernière réforme de l'enseignement du début des années quatre-vingt-dix, on a introduit l'école de base dans la système éducatif tunisien. Elle comporte deux cycles. Le premier cycle qui dure six ans pourrait correspondre à l'ancien enseignement primaire alors que le second cycle qui s'étale sur trois années rappellerait l'enseignement anciennement dispensé dans les collèges. Au total, l'école de base comporte neuf ans d'enseignement au bout desquels les élèves obtiennent le diplôme de fin d'études qui leur permettra alors d'accéder au lycée.

2 L'enseignement au lycée dure quatre ans et permet aux élèves d'obtenir, en fin de cycle, leur baccalauréat.

3 L'enseignement du français commence à partir de la troisième année du premier cycle de l'enseignement de base et dure jusqu'à la fin des études. Avec la dernière réforme, il devient matière obligatoire au même titre que l'arabe.

4 Cette situation de bilinguisme qui a été vécue en réalité par plusieurs générations, constitue un exemple typique d'un mode d'enseignement qui a su réaliser une parfaite synthèse entre la culture arabe et le monde occidental. Le biculturalisme aussi complémentaire que fécond caractérise encore aujourd'hui la spécificité du système éducatif tunisien.

5 On consacre à l'enseignement du français dix heures hebdomadaires dans le premier cycle et trois heures dans les classes de lycées.

\section{Objectifs généraux}

6 L'apprentissage du français dans l'enseignement secondaire contribue à la formation intellectuelle, culturelle et scientifique. C'est aussi un moyen complémentaire pour communiquer avec autrui, pour découvrir d'autres civilisations et cultures et pour 
accéder à l'information scientifique et technique. Le jeune élève tunisien sera ainsi préparé à suivre l'évolution de la culture universelle et à y apporter sa contribution.

$7 \mathrm{Au}$ second cycle de l'enseignement de base, l'élève devra être capable, à l'oral, de communiquer avec autrui, de choisir le registre de langue adapté à la situation de communication dans laquelle il est appelé à s'exprimer ainsi qu'à lire correctement et de façon expressive un texte. Quant au domaine de l'écrit, l'élève devra être capable de produire des énoncés traduisant une connaissance satisfaisante de la langue dans toutes ses composantes : lexicales, syntaxiques, énonciatives... et de pouvoir rédiger plusieurs types de textes (narratifs, descriptifs, argumentatifs...).

Dans les classes du lycée, les objectifs de l'enseignement du français s'articulent autour de trois grandes orientations qui visent à faire prendre conscience aux élèves des caractéristiques du texte littéraire, à perfectionner leurs capacités d'expression personnelle et à développer leur autonomie par l'acquisition de méthodes de travail appropriées. On continue, en outre, à affiner les acquis en grammaire et à les mettre au service des activités de lecture et d'expression, à installer une culture littéraire, à développer l'esprit critique, le sens du relatif et de la nuance.

\section{Méthodes et contenus}

9 Trois grandes activités prédominent dans l'enseignement de base : la lecture, la grammaire et l'expression écrite.

10 Pour la lecture, les compétences visées sont: la lecture proprement dite (courante, correcte et expressive), la compréhension (identification du type de texte, mise en valeur de l'organisation d'ensemble, relevé des informations essentielles, repérage des indices formels de l'énonciation, mise en relief de la signification du texte) et l'appréciation (sensibilisation aux qualités esthétiques du texte, ton, rythme, figures de discours, choix des mots ; prise de conscience du message véhiculé par le texte, morale de l'histoire, valeurs sociales, culturelles...). Quant aux compétences grammaticales, elles consistent à produire des énoncés significatifs et syntaxiquement corrects, à employer correctement les temps et les modes étudiés dans les situations d'énonciation et de communication, à respecter à l'écrit les règles de l'orthographe.

11 Les compétences en expression écrite consistent à rédiger un récit, une description, un dialogue, un récit intégrant une description ou un dialogue, à rédiger un texte argumentatif et à résumer un texte. Tout en maintenant les objectifs de lecture fixés pour les niveaux antérieurs, on développe chez l'élève, dans les classes de lycées, les capacités suivantes: situer le texte par rapport aux grands repères historiques, en dégager la cohérence, rendre compte d'expériences de lecture d'œuvres intégrales.

12 En grammaire, on vise à développer les trois grandes capacités : reconnaître, par l'observation systématique d'énoncés authentiques, les formes et les structures de la langue, analyser le fonctionnement de la phrase, la cohérence et la progression du texte, les formes $\mathrm{du}$ discours, produire, dans des situations authentiques de communication, des énoncés traduisant une appropriation effective du système de la langue. Pour ce qui est de l'apprentissage de l'écrit, il implique le réinvestissement des acquisitions faites par les élèves au cours de toutes les séances de français ainsi que la prise en compte des contraintes spécifiques de l'écrit (grammaire du texte et du discours) par un entraînement systématique et régulier au moyen d'exercices variés, 
passant par les niveaux de la phrase, du texte et du discours. Les objectifs terminaux par année et par capacité s'articulent autour de trois types de compétences: des compétences textuelles générales, des compétences discursives et des compétences méthodologiques consistant en la maitrise des diverses techniques de la production écrite.

\section{Organisation et apprentissage}

Les activités de la classe telles que la lecture, la grammaire et l'expression écrite n'ont pas une fin en elles-mêmes. Par exemple, on n'enseigne pas la grammaire pour la grammaire, néanmoins elle est mise au service de la compréhension des textes et de la production écrite. De même le travail entrepris à propos d'un même texte peut intéresser l'aspect compréhension comme il peut concerner l'aspect production. Cette interdépendance entre les différentes activités de la classe de français se justifie tant sur le plan linguistique que sur les plans didactique et pédagogique.

Le travail est organisé en modules, c'est-à-dire en séquences, sachant que chaque module constitue une unité composée d'une dizaine de séances. Ce mode d'organisation permet de répartir d'une manière équilibrée les contenus et les objectifs d'apprentissage sur l'ensemble de l'année. Trois sortes de modules sont prévues: des modules d'apprentissage, des modules de révision et des modules de lecture.

Le module d'apprentissage s'articulant autour des trois grands types d'activités, lecture, grammaire et expression écrite, peut être envisagé à partir des objectifs spécifiques à chacune d'elle. Afin d'assurer la coordination entre ces matières, on pourrait partir des objectifs discursifs d'expression pour déterminer le contenu du module ou recourir à des supports communs à différentes séances ou établir un lien entre la lecture et l'expression, en entraînant à lire et à produire un même type de texte : le portrait, la narration, l'argumentation... ou réinvestir les acquis des élèves (lexique, structures linguistiques, procédés d'écritures...) dans les différentes activités. Dans chaque module, une séance est réservée à l'évaluation.

16 Le module de révision est conçu dans la perspective d'une pédagogie de soutien qui tient compte des lacunes des élèves et de l'hétérogénéité des classes. Il permet à l'enseignant de poursuivre et d'infléchir son action en vue d'une remise à niveau constante. Son contenu, une batterie d'exercices pertinents, progressifs et variés, est établi par lui à partir d'un corpus d'erreurs relevées à l'occasion des activités d'évaluation.

17 Le module de lecture, enfin, a pour objet l'étude d'une œuvre intégrale. Il intervient deux fois dans l'année, après deux ou trois modules d'apprentissage. Il comporte diverses activités orales et écrites complémentaires: initiation à la lecture et aplanissement des difficultés liées au référent; analyse d'un certain nombre de passages choisis en fonction de leur importance; étude de ses principaux aspects: cadre spatio-temporel, structure narrative ou dramatique, personnages, thèmes dominants... et, enfin, synthèse écrite portant sur telle ou telle problématique. 
Même si la mise en place de la réforme de l'enseignement a bénéficié de beaucoup de conditions favorables : conception d'un programme cohérent et clair, élaboration de manuels adaptés à l'esprit nouveau, présence d'un matériel pédagogique varié, il n'en demeure pas moins que la question de la formation tant initiale que continuée des enseignants reste encore aujourd'hui à préciser. Les enseignants sont libérés un jour par semaine (bien sûr, pas le même pour tout le monde) pour bénéficier de séances de formation ciblées en fonction des besoins, des lacunes constatées et de l'évolution de la didactique de la matière. Cette activité est, en même temps, utile pour la promotion de ces enseignants, pour l'acquisition de la maîtrise de la discipline enseignée et pour le développement de leur compétence professionnelle. Bien sûr, d'autres petits problèmes surgissent ici et là, mais la mission des formateurs de formateurs n'est-elle pas précisément de colmater les brèches et d'entreprendre un travail de terrain, soutenu et pragmatique, difficile certes mais combien exaltant?

\section{RÉSUMÉS}

Le bilinguisme, spécificité du système éducatif tunisien, donne à l'enseignement du français une place de choix permettant aux élèves de réaliser une synthèse entre la culture arabe et le monde occidental. Objectifs, méthodes et contenus font ici l'objet d'une présentation.

\section{INDEX}

Index géographique: Tunisie

Mots-clés : apprentissage du français, enseignement du français, bilinguisme, enseignement primaire, enseignement secondaire

\section{AUTEUR}

\section{AHMED BEN JEMIA}

Inspecteur général de français, ministère de l'Éducation, Tunis, Tunisie 